\title{
The transcription factors Scl and Lmo2 act together during development of the hemangioblast in zebrafish
}

\author{
Lucy J. Patterson, ${ }^{1}$ Martin Gering, ${ }^{2}$ Craig E. Eckfeldt, ${ }^{3}$ Anthony R. Green, ${ }^{4}$ Catherine M. Verfaillie, ${ }^{3}$ Stephen C. Ekker, ${ }^{5}$ \\ and Roger Patient ${ }^{1}$
}

${ }^{1}$ Weatherall Institute of Molecular Medicine, Oxford University, John Radcliffe Hospital, Headington, Oxford, United Kingdom; ${ }^{2}$ Institute of Genetics, University of Nottingham, Queen's Medical Centre, Nottingham, United Kingdom; ${ }^{3}$ Stem Cell Institute and Department of Medicine, University of Minnesota, Minneapolis; ${ }^{4}$ Department of Haematology, Cambridge Institute for Medical Research, Cambridge University, United Kingdom; ${ }^{5}$ Arnold and Mabel Beckman Center for Transposon Research, Department of Genetics, Cell Biology and Development, University of Minnesota, Minneapolis

The transcription factors Scl and Lmo2 are crucial for development of all blood. An important early requirement for $\mathrm{Scl}$ in endothelial development has also been revealed recently in zebrafish embryos, supporting previous findings in $\mathrm{scl}^{-1-}$ embryoid bodies. Scl depletion culminates most notably in failure of dorsal aorta formation, potentially revealing a role in the formation of hemogenic endothelium. We now present evidence that the requirements for Lmo2 in zebrafish embryos are essentially the same as for Scl. The expression of important hemato- poietic regulators is lost, reduced, or delayed, panendothelial gene expression is down-regulated, and aorta-specific marker expression is lost. The close similarity of the phenotypes for $\mathrm{Scl}$ and $\mathrm{Lmo} 2$ suggest that they perform these early functions in hemangioblast development within a multiprotein complex, as shown for erythropoiesis. Consistent with this, we find that $s c l$ morphants cannot be rescued by a non-Lmo2-binding form of Scl but can be rescued by non-DNAbinding forms, suggesting tethering to target genes through DNA-binding part- ners linked via Lmo2. Interestingly, unlike other hematopoietic regulators, the Scl/ Lmo2 complex does not appear to autoregulate, as neither gene's expression is affected by depletion of the other. Thus, expression of these critical regulators is dependent on continued expression of upstream regulators, which may include cell-extrinsic signals. (Blood. 2007;109: 2389-2398)

(C) 2007 by The American Society of Hematology

\section{Introduction}

Lmo2 was first discovered through its homology to the T-cell oncogene $1 \mathrm{~mol}$ and has been found to be activated by chromosomal translocations in several T-cell leukemias. ${ }^{1}$ Like its partner in erythroid cells, $s c l$, during normal development $l m o 2$ is specifically expressed in hematopoietic progenitors, erythroid cells, and megakaryocytes, and in endothelial cells. ${ }^{2,3}$ Targeted gene ablation in mice revealed an essential role for $\mathrm{Lmo} 2$ in primitive hematopoiesis, with $l m o 2^{-1-}$ embryos dying at embryonic day (E) 9 to 10 due to severe anemia. ${ }^{2}$ Lmo $^{-/-}$embryonic stem (ES) cells were unable to contribute to any hematopoietic lineage in chimeras, indicating a critical requirement for $\mathrm{Lmo} 2$ in definitive hematopoiesis. ${ }^{4}$ In addition, although $l m o 2^{-l-}$ ES cells were initially able to contribute to normal primary capillary plexus formation, they were unable to contribute to the formation of larger vessels, and chimeras with a large contribution of $l m o 2^{-1-}$ ES cells exhibited disrupted vascular organization from E9 onwards, indicating a role for $\mathrm{Lmo} 2$ in angiogenesis. ${ }^{3}$

The Lmo2 protein comprises 2 LIM zinc finger-like protein interaction domains and has been found to act as a bridging molecule between the Scl/E47 heterodimer and Gata1/2 in the formation of DNA-binding complexes. ${ }^{5-10}$ A specific phenylalanine residue in helix 2 of the $\mathrm{Scl}$ basic helix-loop-helix (bHLH) domain has been shown to be essential in vitro for hematopoietic activity of Scl. However, rather than being important for heterodimerization

Submitted February 9, 2006; accepted October 25, 2006. Prepublished online as Blood First Edition Paper, November 7, 2006; DOI 10.1182/blood-2006-02003087.

The publication costs of this article were defrayed in part by page charge with E47, this residue promotes protein interactions with Lmo2. ${ }^{11}$ Remarkably, introduction of a phenylalanine residue at the corresponding position in the myogenic bHLH, MyoD, was sufficient to confer hematopoietic activity, rescuing hematopoietic development in $\mathrm{scl}^{-1-} \mathrm{ES}$ cells. The interaction between $\mathrm{Scl}$ and $\mathrm{Lmo} 2$ is therefore essential for initiation of hematopoiesis in ES cells. However, the in vivo requirement for this interaction has not been formally demonstrated. Nevertheless, the fact that both Scl and Lmo2 are similarly required for primitive and definitive hematopoiesis and for angiogenic remodeling of the vasculature suggests that their activities during these processes may be as partners in a multiprotein complex.

Coinjection of $s c l$ and $l m o 2$ mRNA in zebrafish embryos broadens the range of mesodermal tissues that can be converted to a blood and endothelial fate, compared with injection of $s c l$ mRNA alone. ${ }^{12}$ Such gain-of-function experiments suggest that $\mathrm{Scl}$ and Lmo2 may drive hematopoietic and endothelial development through specification of the hemangioblast. If true, one would predict that Lmo2 would turn out to be required for endothelial as well as hematopoietic development in loss-of-function experiments, as found for $\mathrm{Scl}^{13}$

With this in mind, we have examined the role of $1 m o 2$ in hematopoietic and endothelial development by knocking down gene expression using antisense morpholinos in zebrafish embryos. payment. Therefore, and solely to indicate this fact, this article is hereby marked "advertisement" in accordance with 18 USC section 1734. 
In zebrafish, the primitive blood arises from 2 bilateral populations of hemangioblast-like cells within the lateral plate mesoderm of early somite-stage embryos; the anterior lateral-plate mesoderm (ALM), which gives rise to the head vasculature and primitive myeloid cells, and the posterior lateral-plate mesoderm (PLM), which gives rise to the endothelium of the major trunk vessels and a large population of primitive erythrocytes. PLM precursors migrate to the midline, forming the intermediate cell mass (ICM) where primitive erythroid precursors complete their differentiation sandwiched between the forming dorsal aorta (DA) and posterior cardinal vein (PCV). By the end of the first day, a basic vascular loop has been formed and, as the embryonic heart initiates its first contractions, the converged mass of primitive erythrocytes begins to enter circulation. Around this time, runxl staining reveals a row of single hematopoietic stem cells (HSCs) that remain associated with the DA. ${ }^{14}$

Our findings essentially recapitulate those of Scl-depleted zebrafish embryos: primitive and definitive hematopoiesis were lost, and endothelial development was severely disrupted, culminating in loss of the DA. Comparison of the earliest defects in gene expression in $l m o 2$ morphants with those of $s c l$ morphants did not reveal any major differences, implicating Lmo2 as a partner to Scl during this early stage of its activity in hematopoietic and endothelial development. Consistent with this, we show that Lmo2 binding is essential but DNA binding is dispensable for rescue of hematopoiesis and normal endothelial gene expression in $\mathrm{scl}$ morphants. This implies the presence of additional DNA-binding components of the Scl/Lmo2 multiprotein complex.

\section{Materials and methods}

\section{Morpholinos}

Splice morpholinos (MOs) are a particularly powerful method of target gene knockdown because efficacy of splicing inhibition can be directly assessed by reverse transcriptase-polymerase chain reaction (RT-PCR) analysis of the splice products generated.$^{15} \mathrm{Lmo} 2$ functions as a bridging molecule in multiprotein complexes, mediating protein-protein interactions via 2 zinc finger-like LIM domains. ${ }^{16}$ To ensure disruption of these domains, upstream splice sites (ie, the exon 4 splice donor or exon 5 splice acceptor) would have to be targeted (Figure 1A). However, polymorphisms in the intronic sequence at the exon 4 splice donor, and the possibility that in-frame splicing between exons 4 and 6 might occur if the exon 5 splice acceptor was targeted, producing a novel protein with one LIM domain and potentially abnormal function, ruled out either sequence as a good candidate for effective splice MO-mediated gene knockdown. It was therefore not practical to use a splice-blocking approach to knock down Imo 2 expression in zebrafish. We therefore used antisense MOs designed to block $\operatorname{lmo} 2$ translation.

Antisense MOs were obtained from GeneTools (Philomath, OR) with sequences $s c l$ spliceMO 5'-AATGCTCTTACCATCGTTGATTTCA-3', lmo2 atgMO1 5'-CAGTGGCGTTGTTCAATTTCTCCGG-3', and lmo2 $\operatorname{atgMO} 25^{\prime}$-GTAGAAGCCATTTTCAATATGATTC-3'. $10 \mu \mathrm{g} / \mu \mathrm{L}$ and 2.5 $\mu \mathrm{g} / \mu \mathrm{L}$ stock solutions in $\mathrm{dH}_{2} \mathrm{O}$ of $s c l$ spliceMO and lmo2 atgMO1 plus $\mathrm{MO}$ 2, respectively, were prepared and injected into 1- to 16-cell-stage embryos at the yolk/blastomere boundary. Quantities of MOs injected were titrated to appropriate effective nontoxic levels (ie, $6.5 \mathrm{ng} \mathrm{scl}$ spliceMO and $1.75 \mathrm{ng}$ lmo2 atgMO1 and MO2).

\section{mRNA for injection}

Murine $l m o 2$ and zebrafish scl full-length mRNAs were generated as previously described. ${ }^{12} \mathrm{mSCL}$ MH2 and mSCL MH2F constructs ${ }^{11}$ were subcloned by PCR and recombined into a pBUT3 gateway destination vector in front of and in frame with an HA tag. Truncated Scl constructs were made by amplifying sequence encoding Scl bHLH and HLH domains from $\mathrm{pFC}^{17}$ using forward primers $5^{\prime}$-CTCCTCTAGAGCCACCATGGATCCTAGCAATCGAGTCAAGCGC- $3^{\prime}$ and $5^{\prime}$-CTCCTCTAGAGCCACCATGGAGCGCTGGCGACAGCAGAATG- ${ }^{\prime}$, respectively, and reverse primer 5'-CTCCCTCGAGTGGCCCGGGCGGGAGCTTCACC-3'. Products were cloned into pBUT2-MT. ${ }^{17}$ mRNAs were transcribed from linearized templates using mMessage mMachine (Ambion, Huntingdon, United Kingdom). mRNAs were injected, either alone or in combination with $\mathrm{scl} \mathrm{MO}$, into one blastomere of embryos at the 1/2- and occasionally 4-cell stage.

\section{Western analysis}

Whole 6- to 10-somite (s)-stage embryos (15 per lane) were homogenized by vortexing with glass beads (Sigma, Poole, United Kingdom) in $2 \times \mathrm{RSB}$, run on a $4 \%$ to $12 \%$ SDS-polyacrylamide gel, and immunoblotted. Membranes were probed with rat anti-HA (1:1000; Roche, Welwyn Garden City, United Kingdom) followed by antirat (1:2000; Merck Biosciences, Nottingham, United Kingdom) antibodies, or by mouse anti- $\beta$-actin (Clone AC 15; Sigma) followed by antimouse (1:2000; Amersham, Buckinghamshire, United Kingdom) antibodies. Signal was detected by enhanced chemiluminescence (ECL; Amersham).

\section{Whole-mount in situ hybridization}

Breeding zebrafish were maintained and embryos were raised and staged according to Westerfield. ${ }^{18}$ Whole-mount in situ hybridization on zebrafish embryos was carried out as previously described. ${ }^{19}$ Antisense RNAs for in situ hybridization were transcribed from linearized templates using Promega's T3, T7, and SP6 RNA polymerases in the presence of digoxigenin (DIG)-labeled nucleotides (Roche, Burgess Hill, United Kingdom). DIG antibody-alkaline phosphatase conjugate was detected using BM-Purple (Roche) or Fast Red (Sigma). Msr is a novel zebrafish vein-specific marker (IMAGE clone $6795347^{47}$; corresponds to predicted full-length zebrafish clone XM_682265, and shares approximately 65\% protein sequence identity with Xenopus $\mathrm{Msr}^{20}$ and approximately $52 \%$ with mouse Msr, which shows vein-specific expression in the retinal vasculature ${ }^{21}$ ).

\section{Image acquisition and processing}

Embryos were visualized using a Nikon SMZ 1500 zoom stereomicroscope (Nikon, Melville, NY) equipped with a Nikon $1.0 \times$ WD 54 objective lens with an adjustable internal lens ranging from $0.75 \times$ to $11.25 \times$ magnification. Embryos were viewed at 9 to $11.25 \times$ magnification, depending on size. Images were obtained using a Nikon DXM 1200 digital camera and Nikon ACT-1 software (version 2.12) and processed with Adobe Photoshop CS software, version 8.0 (Adobe Systems, San Jose, CA).

\section{Results}

\section{Lmo2-depleted zebrafish embryos fail to develop primitive or definitive blood, phenocopying the $I m o 2^{-I-}$ mouse}

We used antisense MOs designed to block lmo2 translation ("Materials and methods"). The most effective strategy, as assessed by effect on downstream gene expression and incidence of nonspecific defects, entailed injection of 2 MOs, the first overlapping the $\operatorname{lmo} 2 \mathrm{atg}$, and the second binding within the 5' untranslated region (UTR) approximately $300 \mathrm{bp}$ upstream of the atg (Figure 1A). Embryos injected with $1.75 \mathrm{ng}$ of each of these MOs developed essentially normally and without nonspecific defects (Figure 1B).

Although lmo2 morphant embryos appeared grossly normal (Figure 1B), primitive erythrocytes were not observed, either in circulation or accumulated elsewhere. Therefore, Lmo2 depletion in zebrafish embryos recapitulates the phenotype of the $l m o 2^{-1-}$ mouse with respect to the loss of primitive blood. ${ }^{2}$ However, unlike the mouse, and although they eventually develop pericardial edemas, the morphant embryos were able to survive for several 


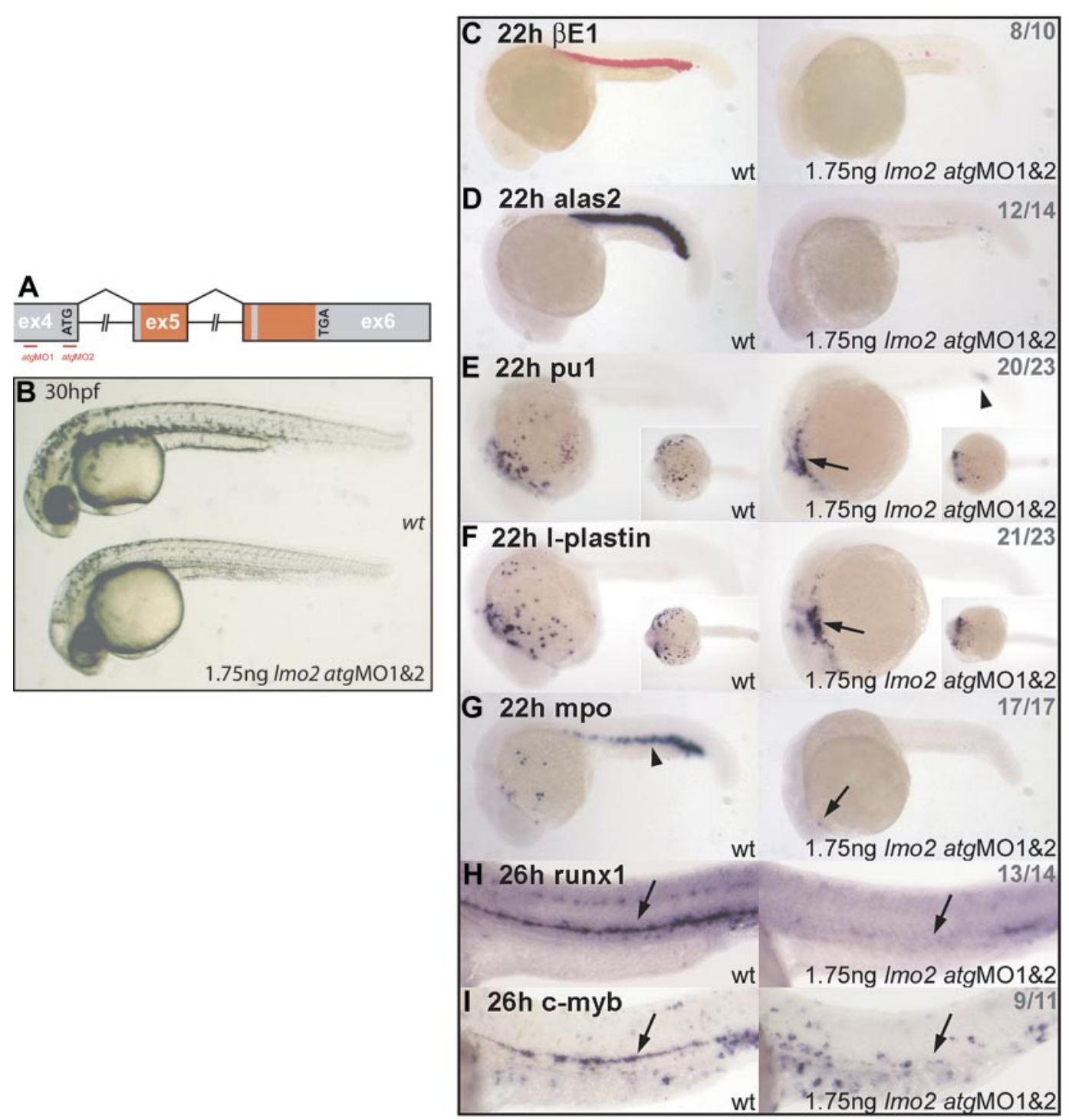

Figure 1. Lmo2 morphants exhibit normal gross morphology but lack hematopoietic gene expression. (A) Genomic structure of the Imo2 gene. Exons (ex) depicted as boxes; atg start and tga stop codons are indicated; intron sizes not to scale; red-shaded regions encode the 2 LIM zinc finger-like domains (the first is split across exons 5 and 6); atgMO binding sites are shown (red lines). (B) Live 30-hpf embryos; lateral views; anterior, left. Lmo2 morphants developed without nonspecific defects. (C-G) Whole-mount embryos; lateral views; anterior, left; inset images in panels $\mathrm{E}$ and $\mathrm{F}$ show ventral view. (H-I) Whole-mount embryos; lateral view; close-up of trunk/tail region; anterior, left. Numbers of embryos represented in gray. (C-D) Erythroid, $\beta E 1$, and alas2 expression is abolished in all morphants at $22 \mathrm{hpf}$. (E-G) In $22-\mathrm{hpf}$ morphants, expression of primitive myeloid genes pu.1, I-plastin, and mpo was severely reduced. Remaining cells were restricted to the heart region (arrows) and, in the case of pu.1, the posterior ICM (arrowhead). (H-I) Expression of HSC markers runx1 and c-myb was significantly down-regulated in the ventral DA of Lmo2-depleted embryos (arrows).

days in the absence of blood circulation. Expression of the primitive erythroid genes beta embryonic globin $1(\beta E 1)^{22}$ and aminolevulinate synthase 2 (alas2), ${ }^{23}$ at 22 hours after fertilization (hpf), was lost in all but a few cells in the ICM (Figure 1C-D). Similarly, expression of pu.1, ${ }^{24}$ 1-plastin, ${ }^{25}$ and myeloperoxidase $(\text { mpo })^{26}$ in primitive myeloid cells was down-regulated in 22-hpf morphants (Figure 1E-G). The nonmotile cells remaining under the heart and in the posterior ICM are likely to be improperly programmed myeloid precursors (Figure 1E-G, arrows; Figure 1E, arrowhead). The mpo expression marking erythroid precursors (Figure 1G, arrowhead) was lost.

Expression of runxl and $c-m y b$ at $26 \mathrm{hpf}$ in the region of the ventral DA, which marks the emerging HSCs, was significantly depleted in morphant embryos (Figure 1H-I, arrows). The loss of expression of these definitive HSC markers in the absence of Lmo2 indicates a failure of definitive hematopoiesis in these embryos, a conclusion also drawn from studies of mouse chimeras where $l m o 2^{-1-}$ ES cells were found to be unable to contribute to any definitive hematopoietic lineage. ${ }^{4}$ However, because the absence of circulation in zebrafish embryos does not confer immediate lethality as it does in the mouse, the failure of DA-associated HSCs to emerge can be observed in Lmo2-depleted embryos as opposed to chimeras.

Several observations indicate that the phenotypes generated by MO injection were specific to knockdown of $l m o 2$. First, morphant embryos developed normally without nonspecific defects, either morphologically (Figure 1B) or in terms of gene expression: nonhematopoietic genes $n k x 2.5^{27}$ in the precardiac mesoderm and pax $2.1^{28}$ in pronephric duct progenitors, tissues that neighbor the developing blood and endothelium, were unperturbed in morphants at the 10s stage (Figure 2A). Similarly, pax2.1 expression in the optic stalk, midbrain/hindbrain boundary region, and otic vesicle was also unaffected by Lmo2 depletion at 10s (Figure 2A, arrows). Secondly, MO-induced defects in hematopoietic and endothelial gene expression were rescued by coinjection with a murine $1 m o 2$ mRNA: the lost expression of gata ${ }^{29}$ and pu. 1 in the PLM, and the reduction in expression of pu. 1 in the ALM and $f t 4^{30}$ in the PLM of 

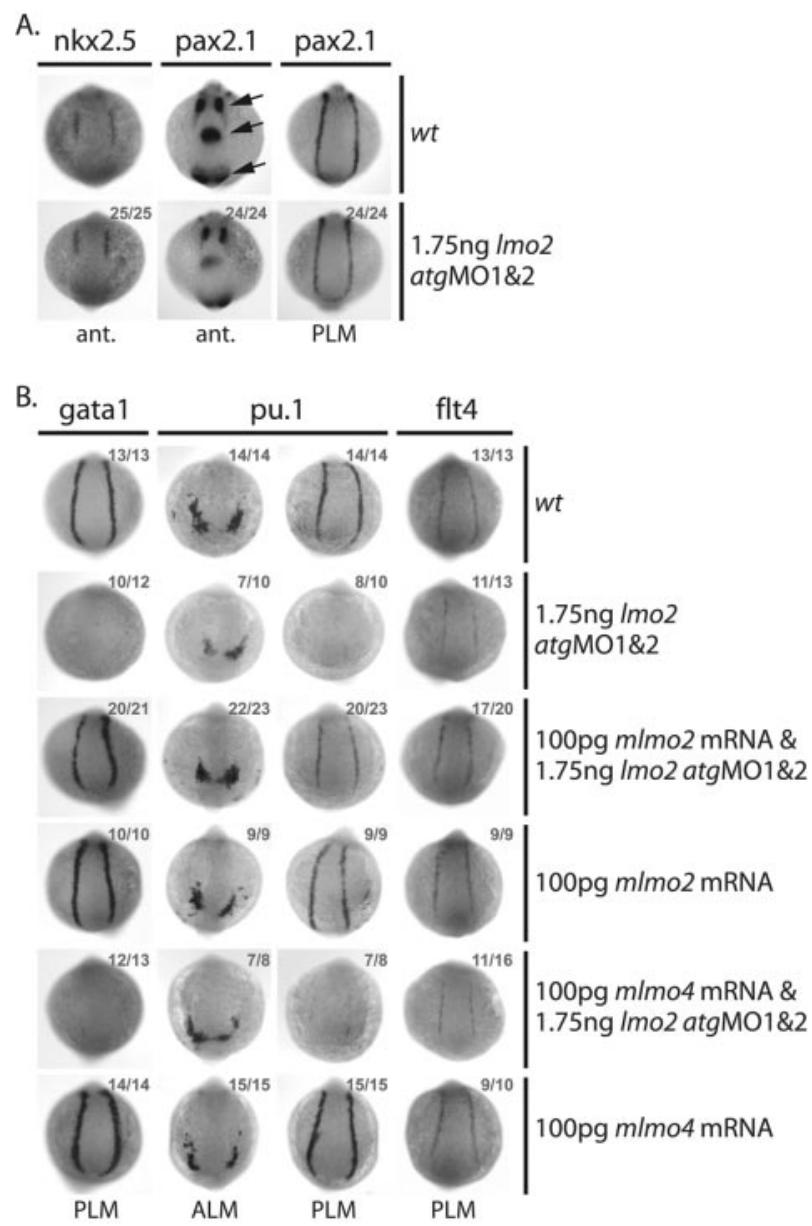

Figure 2. Lmo2 atgMO-mediated knockdown of Lmo2 expression is highly specific. (A-B) Whole-mount 10s embryos; views as indicated; dorsal, top; numbers of embryos represented in gray. (A) Expression of the nonhematopoietic genes $n k x 2.5$ in the precardiac lateral plate mesoderm and pax2.1 in the optic stalk, midbrain/hindbrain boundary region, otic vesicle (arrows, bottom to top), and progenitors of the pronephric duct, immediately adjacent to the primitive erythroid and endothelial precursors in the PLM, was unaffected in morphants. (B) Coinjection of murine $I m o 2$, but not Imo4, mRNA with the Imo2 atgMOs rescued early gata1, pu.1, and flt4 expression levels. 10s embryos was restored to normal levels (Figure 2B). Coinjection of the $l m o 2$ atgMOs with mRNA encoding an alternative member of the Lmo family, murine lmo4, did not restore normal gatal, pu.1, or flt4 expression, confirming specificity of the rescue (Figure 2B). ${ }^{12}$

\section{The position of Lmo2 in the regulatory cascade of hematopoietic genes in the PLM (primitive erythroid and HSCs)}

Precisely where in the regulatory networks leading to blood formation Lmo2 acts was ascertained by analyzing the earliest traceable defects in gene expression in morphant embryos. Lmo2 expression in the PLM initiates between the $2 \mathrm{~s}$ and $3 \mathrm{~s}$ stages, approximately 20 minutes after that of $s c l$ (Figure 3I). Genes expressed in the PLM could be divided into 4 categories with respect to their requirement for $\mathrm{Lmo} 2$.

Dependent: for initiation and maintenance. Expression of the erythroid terminal differentiation genes, $\beta E 1$ globin and alas 2 , and the regulators, gatal, pu.1, and $c-m y b$, was not initiated in $l m o 2$ morphants (Figure 3C-E, I; data not shown). Lmo2 is therefore essential, either directly or indirectly, for the initiation and maintenance of expression of these genes.

Dependent: for timing of initiation. Runxl expression in the PLM of $1 m o 2$ morphants started at the $8 \mathrm{~s}$ stage as opposed to $4 \mathrm{~s}$ in wild-type embryos, and gradually increased (Figure 3B [arrow], I). This suggests that Lmo2 is required for the normal initiation of runxl expression in the PLM, although its expression is subsequently activated and strengthened by other factors. This delay was also seen in scl morphants, albeit not to the same extent as it is in $1 m o 2$ morphants (runxl expression initiated at $6 \mathrm{~s}$ as opposed to $8 \mathrm{~s}$ ). ${ }^{13}$

Dependent: for maintenance. Early broad expression of the zinc-finger transcription factor draculin, ${ }^{25}$ which initiates prior to that of $s c l$ and lmo2, must be independent of both. However, reduction of draculin expression in $\operatorname{lmo} 2$ morphants (ie, Lmo2 dependence), was evident from the $7 \mathrm{~s}$ stage and increased until, at $10 \mathrm{~s}$, draculin expression was lost in all but the most posterior PLM cells around the tailbud (data not shown; Figure 3I). This region of the PLM is both Scl and Lmo2 negative at this time (data not shown), and therefore draculin expression would not be expected to

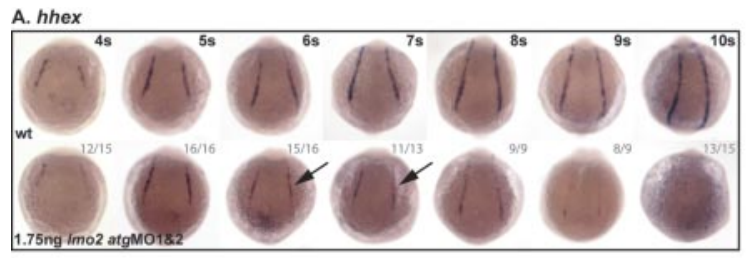

B. runx1
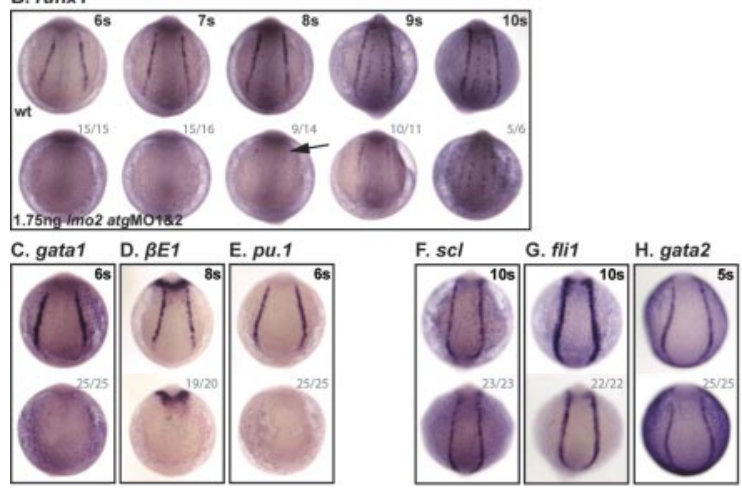

1. Onset of gene expression in the PLM

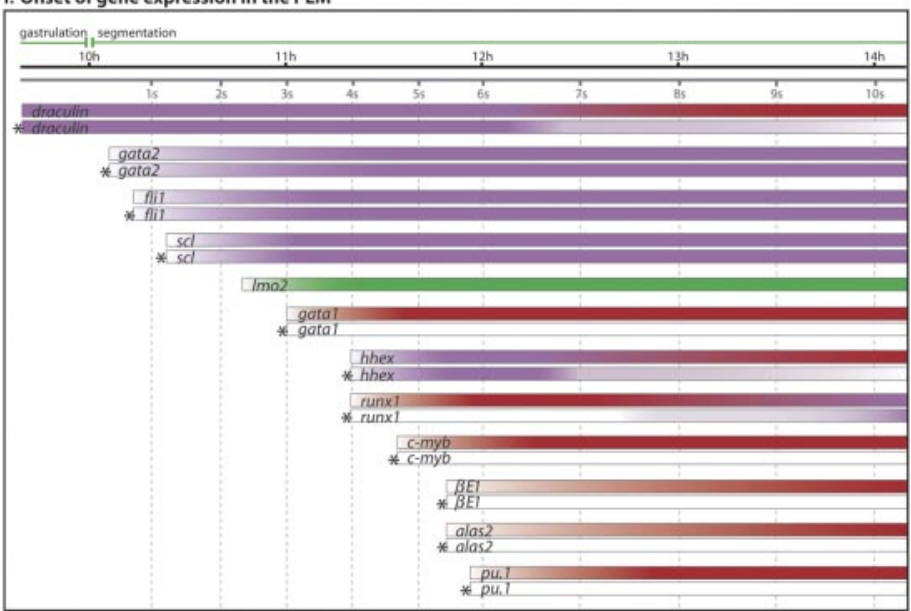

Figure 3. Lmo2 is a critical regulator of PLM development. (A-H) Whole-mount embryos; posterior views; dorsal, top; numbers of embryos represented in gray. (A) Hhex expression initiated as normal, appeared reduced at $6 \mathrm{~s} / 7 \mathrm{~s}$ (arrows) and was lost by $10 \mathrm{~s}$. (B) Runx1 was at first dependent on Scl, initiated weakly in the PLM of morphants at $8 \mathrm{~s}$ (arrow), and increased toward the 10s stage. (C-E) Gata1, $\beta E 1$, and pu. 1 expression was not observed in the PLM of morphant embryos. (F-H) Scl, fli1 and gata2 expression in the PLM was unaffected in 10 s morphants. (I) Data from in situ analyses are summarized schematically. Bars represent the changing expression of genes over time. ${ }^{*}$ Gene expression in morphants. Purple indicates Lmo2 independence; red, Lmo2 dependence; and green, Imo2 expression. 
be $\mathrm{Scl}$ or Lmo2 dependent. This category also includes hhex, ${ }^{31}$ normally expressed in the PLM from around 4s. In lmo2 morphants, hhex expression initiated normally but became reduced from around the $6 \mathrm{~s} / 7 \mathrm{~s}$ stage and was essentially lost by $10 \mathrm{~s}$ (Figure $3 \mathrm{~A}$ [arrows], I). This indicates that while initiation of hhex expression is Lmo2 independent, like draculin, maintenance of hhex in the PLM from around $6 \mathrm{~s} / 7 \mathrm{~s}$ onwards requires Lmo2.

Independent. Genes in this category include putative hemangioblast markers $s c l$, gata2, ${ }^{29}$ and $f i 1,{ }^{30}$ which are expressed in the PLM before $l m o 2$, and must therefore be initiated independently of this transcription factor. The expression of these genes in $1 m o 2$ morphants was unaffected at $10 \mathrm{~s}$ and earlier (Figure 3F-I; data not shown). Thus, a precursor population, expressing hemangioblast genes, was still present in the PLM, although it was unable to elaborate the blood program in the absence of Lmo2.

\section{The position of Lmo2 in the regulatory cascade of hematopoietic genes in the ALM (primitive myeloid)}

As in the PLM, Imo2 is expressed in the ALM from $2 \mathrm{~s}$ to $3 \mathrm{~s}$ (Figure $4 \mathrm{G})$. The consequences of $\mathrm{Lmo} 2$ depletion for the expression of hematopoietic genes in this region fell into the same 4 categories.

Dependent: for initiation and maintenance. Pu.1 and c-myb expression was substantially reduced or lost in the ALM of $1 m o 2$ morphants (Figure 4C, G; data not shown).

Thus, these regulators of myeloid differentiation are dependent, either directly or indirectly, on Lmo2 for their expression.

Dependent: for timing of initiation. Initiation of runxl expression in the ALM was delayed in MO-injected embryos, from 8s (Figure 4B [arrow], G) to 10s (Figure 4B [arrowhead], G), indicating a requirement for $\mathrm{Lmo} 2$ for the correct initiation of runxl expression in the ALM. In this instance, the delay in ALM runxl expression was the same as previously observed in $s c l$ morphants, ${ }^{13}$ indicating an identical requirement for $1 m o 2$ expression.
Dependent: for maintenance. As in the PLM, the broad expression of draculin in the ALM region before $1 m o 2$ is switched on was, as expected, controlled independently of Lmo2. However, by $7 \mathrm{~s}$, when draculin expression is becoming restricted to the ALM, its expression was substantially reduced in morphants, and was lost by $10 \mathrm{~s}$ (data not shown; Figure 4G). In addition, the profile of hhex expression in the ALM of $1 m o 2$ morphants followed that of the PLM with normal expression at $4 \mathrm{~s}$ that decayed by $7 \mathrm{~s}$ and was absent by 10s (Figure 4A [arrows], G). Therefore, as in the PLM, the expression of draculin and hhex in the ALM becomes Lmo2 dependent at $7 \mathrm{~s}$.

Independent. In lmo2 morphants, expression of $s c l$, flil, and gata2 in the ALM initiated normally (Figure 4D-G). Persistence of expression of these early hemangioblast genes indicates that precursor cells were not lost, but their elaboration of the myeloid program was compromised in the absence of Lmo2.

\section{Endothelial gene expression is reduced in Lmo2-depleted embryos}

The expression of endothelial markers was significantly downregulated following injection of the lmo2 atgMOs. While flil expression in the PLM of 10s stage morphants was normal (Figure $3 \mathrm{G}), f k 1^{32}$ and $f t 4$ expression was significantly lower (Figure 5A-B), indicating an important early role for Lmo2 in establishing the program of endothelial development.

At $24 \mathrm{hpf}$, when endothelial precursors have migrated to the midline to form the axial vessels, expression of flil was also reduced, and intersomitic vessels (ISVs) failed to sprout normally (Figure 5C). At $22 \mathrm{hpf}$, expression of tie $1^{33}$ normally delineates the 2 axial vessels, the DA and PCV, separated by a tie $1^{-}$mass of differentiating primitive erythrocytes that have not yet entered circulation. However, staining in $l m o 2$ morphant embryos revealed fewer cells, and these were restricted to the PCV and expressing
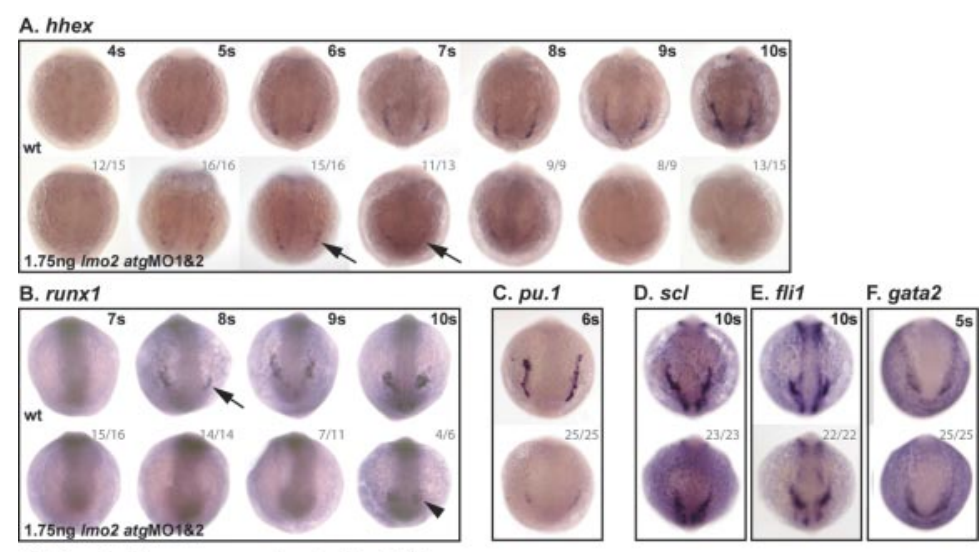

G. Onset of gene expression in the ALM

Figure 4. Lmo2 is a critical regulator of ALM development. (A-F) Whole-mount embryos; posterior views; dorsal, top; numbers of embryos represented in gray. (A) Although staining is generally weak in these embryos, hhex expression initiated as normal, appeared reduced at $6 \mathrm{~s} / 7 \mathrm{~s}$ (arrows) and was lost by 10s. (B) Runx1 was at first dependent on Scl, initiating only weakly in the ALM of morphants at 10 s (arrowhead). Pu.1 expression in the ALM of morphant embryos was substantially reduced (C), while scl, fli1, and gata2 expression was unaffected (D-F). (G) Data from in situ analyses are summarized schematically as previously described for Figure 3.

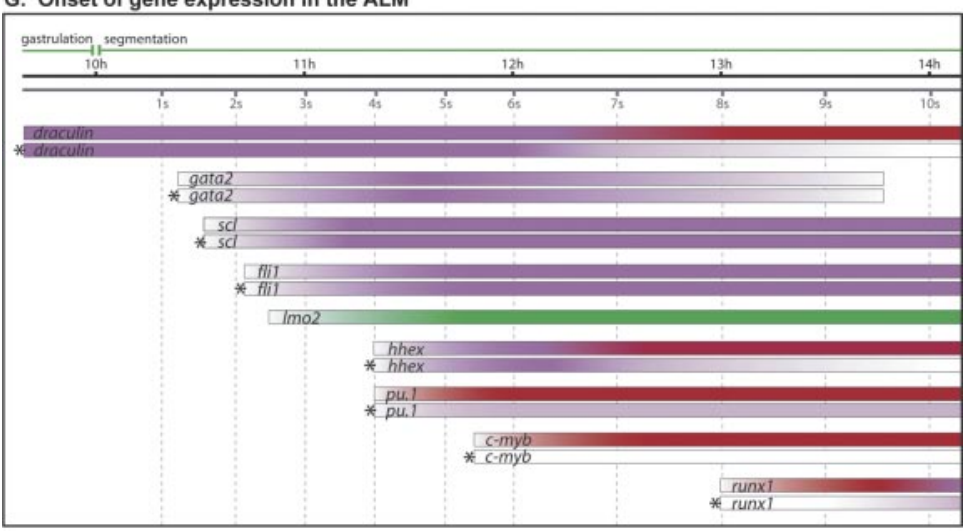


A. $10 \mathrm{~s}$ flk1

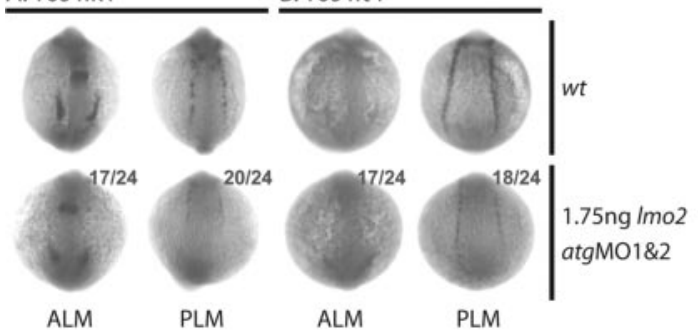

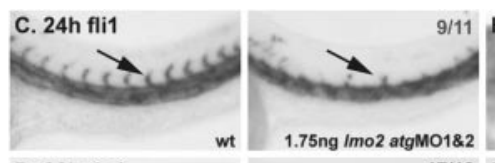

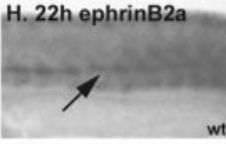

17/18 I. $22 \mathrm{~h}$ deltaC
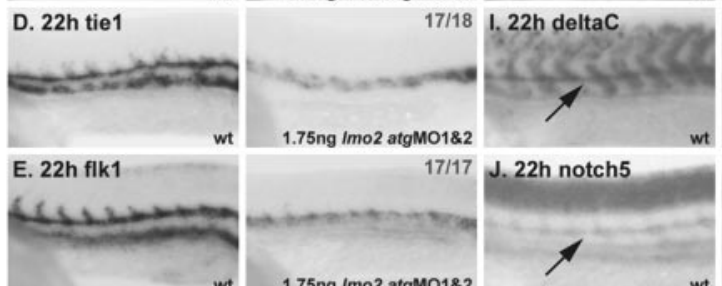

$1.75 \mathrm{ng} / \mathrm{mo} 2$ atgMO1\& $17 / 17$ J. 22h notch5
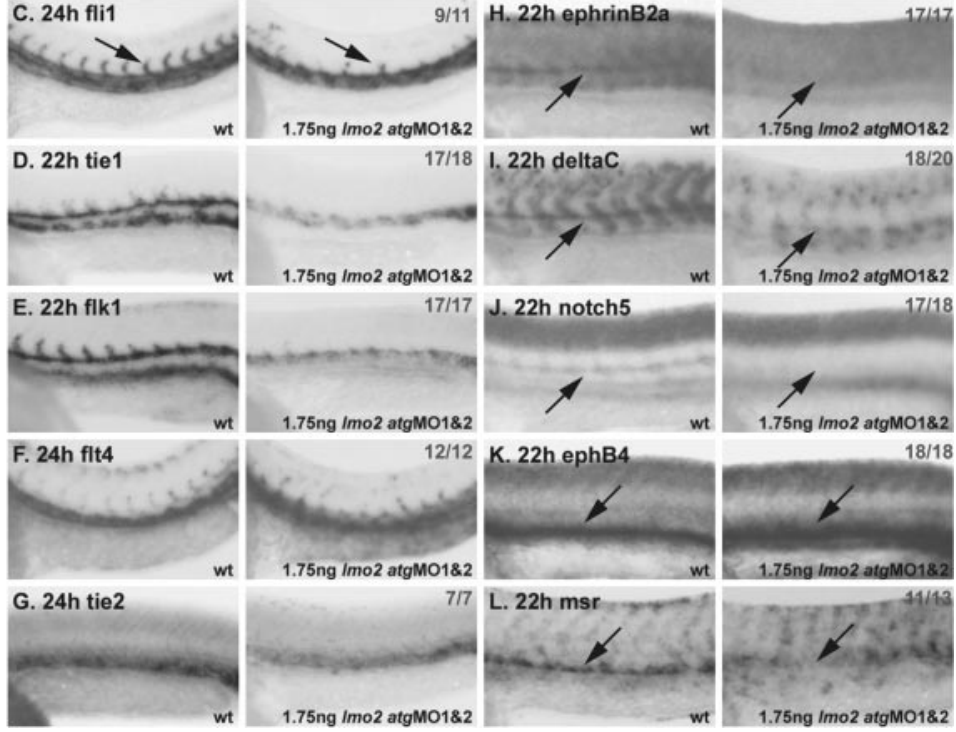

Figure 5. Endothelial development is severely disrupted in Lmo2-depleted embryos. (A-B) Whole-mount 10s embryos; views as indicated; dorsal, top. (C-L) Whole-mount embryos; lateral view; close-up of trunk/tail region; anterior, left. Numbers of embryos represented in gray. (A-B) At 10 s, flk1 and flt4 expression in the ALM and PLM was substantially reduced in morphant embryos. (C) In 24-hpf morphants, fli1 expression was reduced in the trunk region and sprouting of primary ISVs was mostly absent (arrows). (D-E) Tie1 and flk1 expression was significantly reduced in the trunk in 22-hpf morphants. (F-G) However, flt4 and tie2 expression at $24 \mathrm{hpf}$ was not as significantly down-regulated in morphant embryos. (H-J; arrows) Expression of ephrinB2a (H) deltaC (I), and notch5 (J) in the DA was lost in 22-hpf morphants. (K-L; arrows) Although expression of the vein-specific marker ephB4 (K) was not down-regulated, msrexpression in the PCV was lost (L).

tiel only weakly (Figure 5D). The expression of $f k l$, which is usually stronger in the DA than the PCV at this time, was similarly restricted to the PCV in $l m o 2$ morphants (Figure 5E). However, the expression of $f t 4$ and tie $2,{ }^{33}$ which is becoming restricted to the PCV by 24 hpf, was not as significantly reduced in morphants (Figure 5F-G). Furthermore, the expression of artery-specific markers ephrinB2a, ${ }^{34}$ deltaC, ${ }^{34}$ and notch $5^{34}$ in the DA was lost (Figure 5H-J), while vein-specific ephB $4^{34}$ expression in the PCV was not down-regulated (Figure $5 \mathrm{~K}$ ). Together, these data suggest that the DA is more severely affected than the PCV by the depletion of Lmo2. However, although ephB4 expression was not downregulated, PCV development was not entirely normal as residual tiel and $f k 1$ expression in the PCV was reduced (Figure 5D-E). Furthermore, expression of a novel zebrafish vein-specific marker, msr ("Materials and methods"), was also lost (Figure 5L). Therefore, DA development was severely disrupted, and, while PCV endothelial cells were present, their development was also abnormal. This indicates a fundamental requirement for $\mathrm{Lmo} 2$ in endothelial development from the earliest stages.

\section{Lmo2 but not DNA binding by $\mathrm{Scl}$ is required for specification of the primitive erythroid, myeloid and endothelial lineages}

Lmo2 has been shown to interact with $\mathrm{Scl}$ in a multiprotein complex during erythropoiesis. ${ }^{5,6,8-11}$ From the data presented thus far, with 1 minor exception, the molecular phenotype of the lmo2 morphant is indistinguishable from that of the $\mathrm{scl}$ morphant, ${ }^{13}$ suggesting that the early functions of these 2 proteins may also be effected in a multiprotein complex. To test this prediction, we analyzed the requirement for Lmo2 and DNA binding by $\mathrm{Scl}$ in the specification of the primitive erythroid, myeloid, and endothelial lineages. Earlier studies, in $\mathrm{scl}^{-1-} \mathrm{ES}$ cells, demonstrated that the phenylalanine residue in the second helix of Scl, necessary for interaction with $\mathrm{Lmo} 2$, is required to rescue hematopoiesis. ${ }^{11}$ DNA binding, however, is dispensable. ${ }^{35}$ In neither case was endothelial development examined. DNA-binding mutants were also shown to restore both primitive hematopoietic and endothelial gene expression in the zebrafish mutant cloche, which lacks scl expression. However, as these mutants were capable of inducing the expression of endogenous wild-type $s c l$, it was impossible to assess the true Scl DNAbinding requirement for specification of these lineages. This problem is avoided in $\mathrm{scl}$ morphants, because although endogenous $s c l$ expression may be induced, wild-type Scl protein cannot be translated due to aberrant splicing of the $s c l$ pre-mRNA.

As shown previously, ${ }^{13}$ coinjection of wild-type, correctly spliced scl mRNA rescued primitive erythroid (gatal), myeloid (pu.1), and endothelial ( $f t 4)$ gene expression in scl morphants (Figure 6). However, truncated scl constructs consisting of either the bHLH region or the HLH region alone were capable of similar rescues (Figure 6). In addition, the constitutive expression of the truncated Scl proteins in these embryos, just as for wild-type $\mathrm{Scl}$, caused ectopic endothelial gene expression in the paraxial mesoderm. ${ }^{17}$ We therefore conclude that the ability of Scl to direct the differentiation of mesodermal precursors toward a hemangioblast cell fate does not require direct DNA binding by Scl. To determine whether Scl is interacting with Lmo2 when carrying out this function, we made use of the mouse $s c l$ constructs used in the ES cell rescues. ${ }^{11}$ We first tested Scl mutated in the critical phenylalanine residue for the ability to rescue $s c l$ morphant zebrafish embryos. Unfortunately, this protein was not stable in developing embryos and so the failure to rescue could not be assigned to the failure to interact with Lmo2 (data not shown). We therefore tested mSCL MH2, in which the second helix of the mouse Scl protein is substituted with that of the nonhematopoietic bHLH MyoD. While this protein is still able to dimerize with $\mathrm{E} 2 \mathrm{~A}$, it is unable to interact with $\mathrm{Lmo} 2$ and cannot rescue in vitro hematopoiesis from $\mathrm{scl}^{-1-}$ ES cells. In contrast, mSCL MH2F includes a further modification: a single amino acid substitution which reintroduces the critical phenylalanine residue of the Scl second helix, restoring the ability of the construct to interact with Lmo2 and therefore rescue hematopoiesis from $s \mathrm{sl}^{-1-}$ ES cells. When mSCL MH2 and MH2F RNAs were tested for their abilities to rescue scl morphant zebrafish embryos, $\mathrm{MH} 2 \mathrm{~F}$ but not $\mathrm{MH} 2$ was able to rescue gatal, 
Figure 6. Scl DNA binding is not required for early erythroid, myeloid, or endothelial gene expression. Whole-mount $10 \mathrm{~s}$ embryos; posterior/anterior views as indicated; dorsal, top; numbers of embryos represented in gray. Coinjection of sclexpression constructs containing only the bHLH or HLH domains with the $s c l$ spliceMO was sufficient to rescue early gata1 and pu.1 expression in the PLM and the PLM and ALM, respectively. Expression of the endothelial gene flt4 in the PLM could also be restored to normal levels, and ectopic expression of full-length $s c l$ or either construct resulted in the expansion of flt4 expression into the presomitic mesoderm (arrowheads). ${ }^{12,17}$ Structures of Scl constructs used in each case are illustrated.

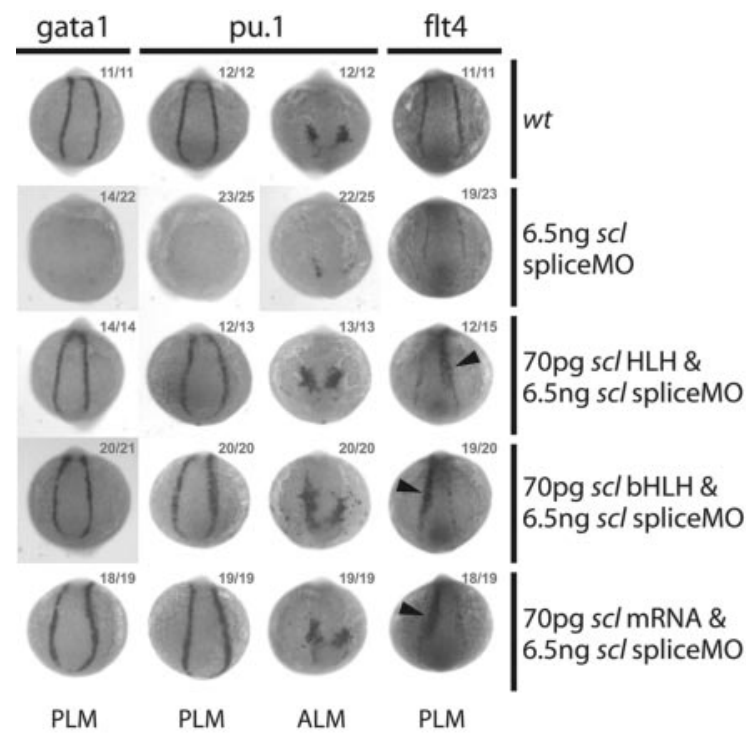

Structure of $s \mathrm{Cl}$ constructs injected:

HLH

\section{HLH}

pu.1, and flt expression, representing primitive erythroid, myeloid, and endothelial lineages (Figure 7A). Western analysis showed that similar amounts of protein had been synthesized in the injected embryos (Figure 7B). We therefore conclude that $\mathrm{Scl}$ and Lmo2 act together in a multiprotein complex to induce hemangioblasts in zebrafish embryos.

\section{Discussion}

\section{Lmo2 is essential for hematopoiesis}

MO-mediated knockdown of $1 m o 2$ expression in zebrafish effectively recapitulated the gross hematopoietic defects of the $l m o 2^{-/-}$ mouse. Morphant embryos developed without primitive or definitive blood: primitive erythroid gene expression was essentially lost, primitive myeloid gene expression was significantly reduced, and the expression of genes associated with definitive hematopoiesis in DA-associated HSCs was mostly absent.

Just as lmo2-null mouse embryos display the same gross hematopoietic defects as those with targeted deletions in $s c l,{ }^{2,4}$ the effects on hematopoietic gene expression seen following injection of the $\operatorname{lmo} 2$ atgMOs were essentially the same as those previously obtained in $\mathrm{scl}$ morphants. ${ }^{13}$ Expression of flil and gata2, which initiates prior to that of both $\mathrm{scl}$ and $\mathrm{lmo} 2$ and was normal in both morphants, is therefore $\mathrm{Scl}$ and $\mathrm{Lmo} 2$ independent. The direct or indirect requirements for Lmo2 for expression of all other hematopoietic and endothelial genes analyzed were essentially the same as the requirements for $\mathrm{Scl},{ }^{13}$ strongly suggesting that $\mathrm{Scl}$ and $\mathrm{Lmo} 2$ act together within a multiprotein complex during development and differentiation of the hemangioblast. The only observed exception to this was a subtle difference in the control of runxl expression in the PLM. Runxl expression, in the absence of Scl, began to recover at an earlier timepoint than in the absence of Lmo2. This is likely to reflect a genuine difference between control by $\mathrm{Scl}$ and $\mathrm{Lmo} 2$ rather than a difference in penetrance of MOs, as the delay in runxl expression in the ALM was the same in both morphants. It is possible that Lmo2 may interact and collaborate with as yet unknown factors that rescue expression of runxl in the PLM in the absence of Scl, enabling expression of runxl at an earlier timepoint than is possible in the absence of Lmo2.
A.

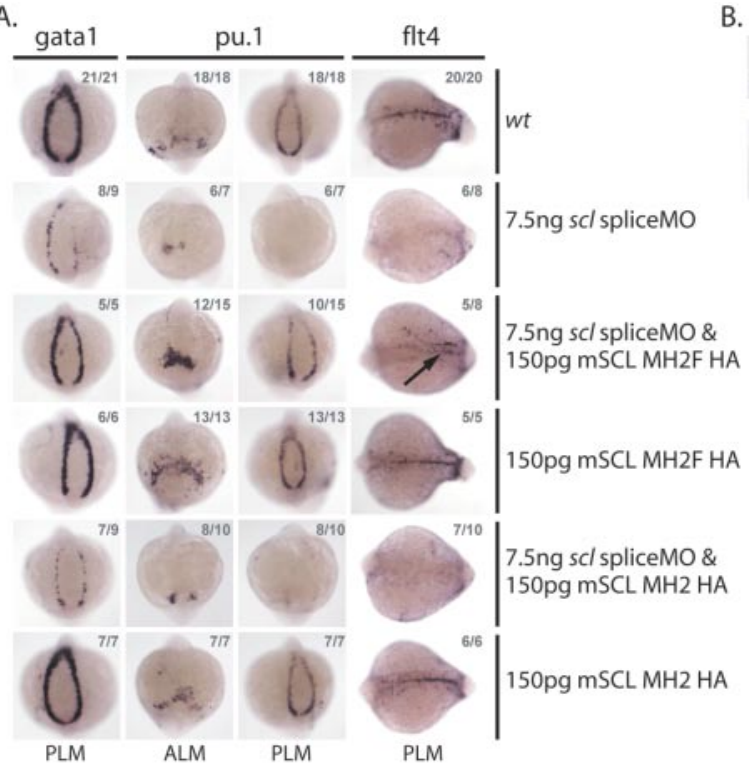

B.

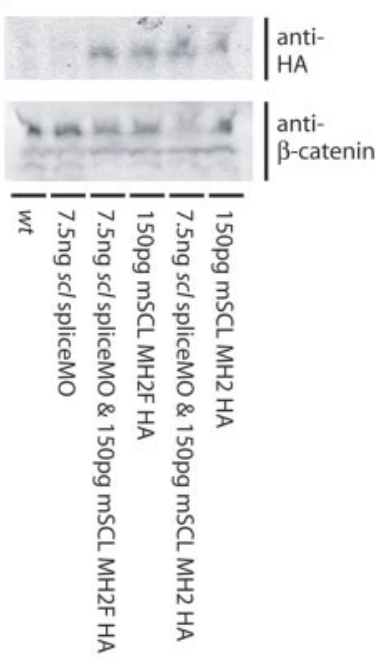

Figure 7. Lmo2 binding is required for early erythroid, myeloid, and endothelial gene expression. (A) Erythroid (gata 1 and pu. 1 in the PLM), myeloid (pu. 1 in the ALM), and endothelial (flt4) gene expression defects in $s c /$ morphants can only be rescued by coinjection with an mRNA encoding a bHLH construct which includes the phenylalanine residue of the Scl second helix essential for the interaction between Scl and Lmo2. Whole-mount $15 \mathrm{~s}$ embryos; views as indicated; numbers of embryos represented in gray. Arrow indicates rescued endothelial gene expression in the position of the dorsal aorta. (B) Western analysis shows that both HA-tagged constructs are expressed in injected whole-embryo extracts. 


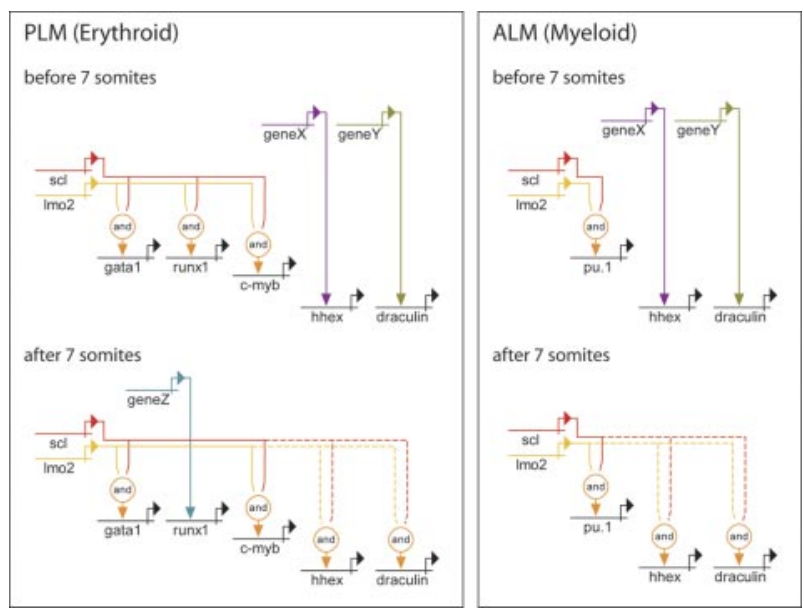

Figure 8. Genetic regulatory networks controlling early PLM and ALM development. Direct relationships, illustrated by continuous lines, are defined by 3 criteria: (1) target gene expression is affected by perturbation of activator; (2) target gene and activator are coexpressed; (3) target gene promoter/enhancer sequences contain binding sites for activators, or length of time between perturbation of activator and effect on target gene is probably insufficient to allow for synthesis of intermediates. Where criteria 1 and 2 are met but 3 is unknown, the relationship is described as indirect and depicted by a dashed line. PLM (erythroid): the data show that initiation of gata1 and c-myb expression is both Scl and $\mathrm{Lmo2}$ dependent. Initially, runx1 expression is dependent on $\mathrm{Scl}$ and Lmo2, while hhex and draculin are $\mathrm{Scl}$ and $\mathrm{Lmo2}$ independent. After 7s, hhex and draculin become dependent on $\mathrm{Scl}$ and $\mathrm{Lmo2}$, and runx1 expression gradually becomes $\mathrm{Scl}$ and Lmo2 independent. ALM (myeloid): pu.1 expression is initially dependent on Scl and Lmo2, while hhex and draculin are independent. After 7s, hhex and draculin become $\mathrm{Scl}$ and $\mathrm{Lmo} 2$ dependent. Unknown activators of Scl- and Lmo2independent genes are depicted as genes $\mathrm{X}, \mathrm{Y}$, and $\mathrm{Z}$. It is possible that $\mathrm{X}$ and $\mathrm{Y}$ represent the same gene due to similarities in timing of involvement.

In our previous study we used the length of time between the first appearance of $\mathrm{scl}$ expression and that of its target genes to determine the likelihood of those targets being directly activated by Scl. ${ }^{13}$ Candidate direct targets included gatal and runxl in the PLM and pu.1 in the ALM. As $1 m o 2$ expression initiates approximately 20 minutes later than that of $s c l$ in both the PLM and ALM, there would be even less time between the assembly of a fully functional Scl/Lmo2 complex and activation of target genes for the generation of intermediates. Lmo2 expression initiates approximately 20 minutes before that of gatal in the PLM, 30 minutes before that of pu. 1 in the ALM, and 30 minutes before that of runxl in the PLM. This shorter period therefore increases the likelihood that expression of gatal, pu.1, and runxl is directly activated by the Scl/Lmo2 multiprotein complex (Figure 8). It is also possible that early PLM expression of $c-m y b$, a proto-oncogene believed to play a role in controlling the proliferation and differentiation of HSCs, ${ }^{36}$ is activated directly by the $\mathrm{Scl} / \mathrm{Lmo} 2$ complex as its expression initiates around 40 minutes after that of $\operatorname{lmo} 2$ (Figure 8). This is the first time that a direct role for the $\mathrm{Scl} / \mathrm{Lmo} 2$ complex in regulation of $c-m y b$ expression has been suggested.

Again, just as in $s c l$ morphants, ${ }^{13}$ hhex and draculin expression in both the PLM and ALM was initially Lmo2 independent, but from the $7 \mathrm{~s}$ stage onwards became progressively reduced and therefore increasingly dependent on Lmo2 for maintenance (Figure 8). The delay in onset of runxl expression in both $l m o 2$ and $\mathrm{scl}$ morphants ${ }^{13}$ indicates that it is initially dependent on activation by the $\mathrm{Scl} / \mathrm{Lmo} 2$ multiprotein complex, but is subsequently driven by alternative factors (Figure 8).

To conclude, the defects in gene expression in the PLM and ALM in both morphants imply critical early roles for Lmo2 and Scl within a multiprotein complex that functions at or near the top of the hierarchy of transcription factors governing hematopoietic development in both populations.

\section{Lmo2 and endothelial development}

The endothelial defects generated on injection of the $1 m o 2$ atgMOs were the same as those seen in $\mathrm{scl}$ morphants with respect to the genes monitored. ${ }^{13}$ Early expression of $f l k 1$ and $f l t 4$ was reduced in endothelial precursors, indicating an early requirement for Lmo2 in the endothelial program. Later, panendothelial gene expression in the trunk region was reduced, ISV formation was mostly absent, and DA-specific gene expression was lost. These observations support a role for $\mathrm{Lmo}$, alongside $\mathrm{Scl}$, in vascular endothelial growth factor (VEGF) signaling through maintenance of $f k 1$ expression. This early dependence on the Scl/Lmo2 multiprotein complex for correct $f k l$ expression, and the resulting severe early endothelial defects, may be peculiar to zebrafish where $s c l$ and lmo 2 expression initiates before that of $f k 1$. However, in mouse embryos, although $f l k l$ is expressed prior to and independently of $s c l$, there is evidence to suggest a role for Scl, and presumably therefore the $\mathrm{Scl} / \mathrm{Lmo} 2$ multiprotein complex, in maintenance of flkl expression. ${ }^{37-39}$ In doing so, the Scl/Lmo2 complex may still play a role in mice in the development of hemogenic endothelium at the time of HSC emergence in the aorta-gonad-mesonephros (AGM). Consistent with this notion, expression of both genes is elevated in the DA at this time. ${ }^{40}$

As in $s c l$ morphants, ephB4 expression in the PCV in $l m o 2$ morphants was normal, while $m s r$ expression was lost, suggesting that PCV development is also compromised when $\mathrm{Lmo} 2$ or $\mathrm{Scl}$ is depleted. As PCV development is essentially normal when VEGF signaling alone is disrupted, there must be an extra requirement for the Scl/Lmo2 complex during venous endothelial development over and above its role in VEGF signaling. This comparatively lesser and VEGF-independent role in endothelial development may be analogous to the late angiogenic defects observed in $\mathrm{scl}^{-/-}$and lmo $2^{-1-}$ mouse embryos..$^{3,41-43}$

\section{Lmo2 and Scl are obligate partners}

The close molecular similarity of the morphant phenotypes for lmo 2 and $s c l$ raised the possibility that they might be working together in a complex, as has been shown during erythropoiesis. , $, 6,8-11$ Consistent with this suggestion, we found that Scl's function could not be carried out by a mutant version unable to bind Lmo2 although a non-DNA binding mutant was still active, implying a critical requirement for the interaction between $\mathrm{Scl}$ and $\mathrm{Lmo} 2$ and the presence of cofactors that tether them to the DNA, allowing activation of target genes. Lmo2 has been shown to bind Scl via specific residues in the second helix, bridging an interaction between the Scl/E47 heterodimer and either GATA1 or GATA2. ${ }^{11}$ It seems, therefore, that the presence of Lmo2 is essential for activation of Scl target genes in the absence of Scl DNA binding to mediate the interaction between $\mathrm{Scl}$ and the DNA-binding subunits of the complex. However, in addition, MO-mediated knockdown of lmo2 expression in zebrafish embryos strongly suggests that this interaction is essential for activation of Scl target genes even when Scl DNA-binding activity is intact. The DNA-binding mutant $\mathrm{Scl}$ data recapitulate previous studies, both in vitro in ES cells and in vivo in zebrafish embryos, in which $\mathrm{Scl}$, but not direct DNA binding by $\mathrm{Scl}$, was shown to be required for the specification of the primitive hematopoietic lineages. ${ }^{35,44}$ Also, for the first time, we show that Scl's role in normal endothelial development, potentially at the level of the hemangioblast, does not require DNA binding. The data available, therefore, strongly suggest that $\mathrm{Scl}$ and Lmo2 are obligate partners within a multiprotein complex that drives development and differentiation of the hemangioblast. 


\section{Is positive feedback required for maintenance of $s c /$ and Imo2 expression?}

Positive feedback loops and autoregulation are common features of genetic regulatory networks, important for stabilizing expression of key regulators and driving the forward momentum of differentiation (Howard and Davidson ${ }^{45}$ and references therein). Indeed, such relationships are frequently observed in the regulatory networks that drive hematopoiesis, with gatal, gata2, fli1, pu.1, and c-myb all using autoregulation to maintain their expression (Swiers et $\mathrm{al}^{46}$ and references therein). However, identifying autoregulation in MO studies can be misleading: target gene RNAs are likely to be degraded either because they are defective or because they are not on polysomes. However, since the evidence strongly suggests that $\mathrm{Scl}$ and Lmo2 activate target genes as obligate partners, disruption of that partnership through MO-mediated knockdown of one component will indirectly reveal the requirement for positive feedback in maintaining gene expression of the other. Thus, at least until the $10 \mathrm{~s}$ stage, $\mathrm{scl}$ expression was unperturbed in $l m o 2$ morphants and must therefore be Lmo2 independent, just as $1 m o 2$ expression in $\mathrm{scl}$ morphants was normal and therefore $\mathrm{Scl}$ independent. Since loss of either component appears to be sufficient to abolish the activity of the entire complex, it seems unlikely that positive feedback by the Scl/Lmo2 complex is involved in maintain- ing the levels of $\mathrm{Scl}$ and $\mathrm{Lmo}$, at least during early development and differentiation of the hemangioblast.

\section{Acknowledgments}

We would like to thank C. Porcher for mSCL MH2 and MH2F constructs, and J. Campos-Ortega, Y. Cheng, K. Crosier, M. Fishman, M. Hammerschmidt, P. Ingham, G. Lieschke, K. Peters, A. Rodaway, D. Stainier, S. Wilson, and L. Zon for probes.

Supported by the Medical Research Council (MRC) (R.P. and M.G.), the Biotechnology and Biological Sciences Research Council (BBSRC) (L.J.P.), and National Institutes of Health (NIH) grants PO1-CA-65493 (C.M.V. and C.E.E.) and GM63904 (S.C.E.).

\section{Authorship}

Conflict-of-interest statement: The authors declare no competing financial interests.

Correspondence: Roger Patient, Weatherall Institute of Molecular Medicine, Oxford University, John Radcliffe Hospital, Headington, Oxford, OX3 9DS United Kingdom; e-mail: roger. patient@imm.ox.ac.uk.

\section{References}

1. Rabbitts TH. LMO T-cell translocation oncogenes typify genes activated by chromosomal translocations that alter transcription and developmental processes. Genes Dev. 1998;12:2651-2657.

2. Warren $\mathrm{AJ}$, Colledge $\mathrm{WH}$, Carlton MBL, Evans MJ, Smith AJH, Rabbitts TH. The oncogenic cysteine-rich lim domain protein rbtn2 is essential for erythroid development. Cell. 1994;78:45-57.

3. Yamada Y, Pannell R, Forster A, Rabbitts TH. The oncogenic LIM-only transcription factor Lmo2 regulates angiogenesis but not vasculogenesis in mice. Proc Natl Acad Sci U S A. 2000;97:320324.

4. Yamada Y, Warren AJ, Dobson C, Forster A, Pannell R, Rabbitts TH. The T cell leukemia LIM protein $\mathrm{Lmo} 2$ is necessary for adult mouse hematopoiesis. Proc Natl Acad Sci U S A. 1998;95: 3890-3895.

5. Osada H, Grutz G, Axelson H, Forster A, Rabbitts $\mathrm{TH}$. Association of erythroid transcription factors: complexes involving the LIM protein RBTN2 and the zinc-finger protein GATA-1. Proc Natl Acad Sci U S A. 1995;92:9585-9589.

6. Wadman IA, Osada H, Grutz GG, et al. The LIMonly protein Lmo2 is a bridging molecule assembling an erythroid, DNA-binding complex which includes the TAL1, E47, GATA-1 and Ldb1/NL1 proteins. EMBO J. 1997;16:3145-3157.

7. Lecuyer E, Herblot S, Saint-Denis M, et al. The SCL complex regulates c-kit expression in hematopoietic cells through functional interaction with Sp1. Blood. 2002;100:2430-2440.

8. Xu Z, Huang S, Chang LS, Agulnick AD, Brandt SJ. Identification of a TAL1 target gene reveals a positive role for the LIM domain-binding protein Ldb1 in erythroid gene expression and differentiation. Mol Cell Biol. 2003;23:7585-7599.

9. Lahlil R, Lecuyer E, Herblot S, Hoang T. SCL assembles a multifactorial complex that determines glycophorin A expression. Mol Cell Biol. 2004;24: 1439-1452.

10. Anguita E, Hughes J, Heyworth C, Blobel GA, Wood WG, Higgs DR. Globin gene activation during haemopoiesis is driven by protein complexes nucleated by GATA-1 and GATA-2. EMBO J. 2004;23:2841-2852.

11. Schlaeger TM, Schuh A, Flitter S, et al. Decoding hematopoietic specificity in the helix-loop-helix domain of the transcription factor SCL/Tal-1. Mol Cell Biol. 2004;24:7491-7502.

12. Gering $M$, Yamada $Y$, Rabbitts $T H$, Patient RK. Lmo2 and Scl/Tal1 convert non-axial mesoderm into haemangioblasts which differentiate into endothelial cells in the absence of Gata1. Development. 2003;130:6187-6199.

13. Patterson LJ, Gering M, Patient R. Scl is required for dorsal aorta as well as blood formation in zebrafish embryos. Blood. 2005;105:3502-3511.

14. Gering M, Patient R. Hedgehog signaling is required for adult blood stem cell formation in zebrafish embryos. Dev Cell. 2005;8:389-400.

15. Draper BW, Stock DW, Kimmel CB. Zebrafish fgf24 functions with fgf8 to promote posterior mesodermal development. Development. 2003;130: 4639-4654.

16. Nam $\mathrm{CH}$, Rabbitts $\mathrm{TH}$. The role of $\mathrm{LMO} 2$ in development and in T cell leukemia after chromosomal translocation or retroviral insertion. Mol Ther. 2006;13:15-25.

17. Gering M, Rodaway ARF, Gottgens B, Patient RK, Green AR. The SCL gene specifies haemangioblast development from early mesoderm. EMBO J. 1998:17:4029-4045.

18. Westerfield M. The Zebrafish Book: A Guide for the Laboratory use of Zebrafish (Brachydanio rerio). Eugene, OR: University of Oregon Press; 1993.

19. Jowett $T$, Yan YL. Double fluorescent in situ hybridization to zebrafish embryos. Trends Genet. 1996;12:387-389.

20. Devic E, Paquereau L, Vernier P, Knibiehler B, Audigier Y. Expression of a new G-coupled receptor $\mathrm{X}$-msr is associated with an endothelial lineage in Xenopus laevis. Mech Dev. 1996;59:129140.

21. Saint-Geniez M, Argence CB, Knibiehler B, Audigier $Y$. The msr/apj gene encoding the apelin receptor is an early and specific marker of the venous phenotype in the retinal vasculature. Gene Expr Patterns. 2003;3:467-472.

22. Quinkertz A, Campos-Ortega JA. A new beta-globin gene from the zebrafish, beta(E1), and its pattern of transcription during embryogenesis. Dev Genes Evol. 1999;209:126-131.

23. Brownlie A, Donovan A, Pratt SJ, et al. Positional cloning of the zebrafish sauternes gene: a model for congenital sideroblastic anaemia. Nat Genet. 1998;20:244-250.

24. Lieschke GJ, Oates AC, Paw BH, et al. Zebrafish SPI-1 (PU.1) marks a site of myeloid development independent of primitive erythropoiesis: implications for axial patterning. Dev Biol. 2002;246: 274-295.

25. Herbomel P, Thisse B, Thisse C. Ontogeny and behaviour of early macrophages. Development. 1999;126:3735-3745.

26. Bennett CM, Kanki JP, Rhodes J, et al. Myelopoiesis in the zebrafish, Danio rerio. Blood. 2001;98: 643-651.

27. Chen J-N, Fishman MC. Zebrafish tinman homolog demarcates the heart field and initiates myocardial differentiation. Development. 1996; 122:3809-3816.

28. Pfeffer PL, Gerster T, Lun K, Brand M, Busslinger $M$. Characterization of three novel members of the zebrafish Pax2/5/8 family: dependency of Pax5 and Pax8 expression on the Pax2.1 (noi) function. Development. 1998;125:3063-3074.

29. Detrich HW, Kieran MW, Chan FY, et al. Intraembryonic hematopoietic cell migration during vertebrate development. Proc Nat Acad Sci U S A. 1995;92:10713-10717.

30. Thompson MA, Ransom DG, Pratt SJ, et al. The cloche and spadetail genes differentially affect hematopoiesis and vasculogenisis. Dev Biol. 1998;197:248-269.

31. Liao W, Ho C-Y, Yan YL, Postlethwait J, Stainier DYR. Hhex and Scl function in parallel to regulate early endothelial and blood differentiation in zebrafish. Development. 2000;127:4303-4313.

32. Fouquet B, Weinstein BM, Serluca FC, Fishman $M C$. Vessel patterning in the embryo of the zebrafish: guidance by notochord. Dev Biol. 1997; 183:37-48.

33. Lyons MS, Bell B, Stainier D, Peters KG. Isolation of the zebrafish homologues for the tie-1 and tie-2 endothelium-specific receptor tyrosine kinases. Dev Dyn. 1998;212:133-140.

34. Lawson ND, Scheer N, Pham VN, et al. Notch signaling is required for arterial-venous differentiation during embryonic vascular development. Development. 2001;128:3675-3683. 
35. Porcher C, Liao EC, Fujiwara Y, Zon LI, Orkin SH. Specification of hematopoietic and vascular development by the bHLH transcription factor SCL without direct DNA binding. Development. 1999; 126:4603-4615

36. Sandberg ML, Sutton SE, Pletcher MT, et al. CMyb and p300 regulate hematopoietic stem cell proliferation and differentiation. Dev Cell. 2005;8: 153-166.

37. Ema M, Faloon P, Zhang WJ, et al. Combinatorial effects of Flk1 and Tal1 on vascular and hematopoietic development in the mouse. Genes Dev. 2003; 17:380-393.

38. Kappel A, Schlaeger TM, Flamme I, Orkin SH Risau W, Breier G. Role of SCL/Tal-1, GATA, and ets transcription factor binding sites for the regulation of flk-1 expression during murine vascular development. Blood. 2000;96:3078-3085

39. Hirai H, Ogawa M, Suzuki N, et al. Hemogenic and nonhemogenic endothelium can be distinguished by the activity of fetal liver kinase (Flk)-1 promoter/enhancer during mouse embryogenesis. Blood. 2003;101:886-893.

40. Jaffredo T, Bollerot K, Sugiyama D, Gautier R, Drevon C. Tracing the hemangioblast during em bryogenesis: developmental relationships between endothelial and hematopoietic cells. Int $J$ Dev Biol. 2005;49:269-277.

41. Robb L, Lyons I, Li RL, et al. Absence of yolk-sac hematopoiesis from mice with a targeted disruption of the SCL gene. Proc Natl Acad Sci U S A. 1995;92:7075-7079.

42. Elefanty AG, Begley CG, Hartley L, Papaevangeliou $B$, Robb L. SCL expression in the mouse embryo detected with a targeted lacZ reporter gene demonstrates its localization to hematopoietic, vascular, and neural tissues. Blood. 1999;94 3754-3763.
43. Visvader JE, Fujiwara Y, Orkin SH. Unsuspected role for the T-cell leukemia protein SCL/tal-1 in vascular development. Genes Dev. 1998;12:473479.

44. Juarez MA, Su F, Chun S, Kiel MJ, Lyons SE. Distinct roles for SCL in erythroid specification and maturation in zebrafish. J Biol Chem. 2005; 280:41636-41644

45. Howard ML, Davidson EH. cis-regulatory contro circuits in development. Dev Biol. 2004;271:109 118.

46. Swiers G, Patient R, Loose M. Genetic regulatory networks programming haematopoietic stem cells and erythroid lineage specification. Dev Biol. 2006:294:525-540.

47. Lennon GG, Auffray C, Polymeropoulous M, Soares MB. The I.M.A.G.E. consortium: an integrated molecular analysis of genomes and their expression. Genomics. 1996;33:151-152. 